\title{
Influence of electric pulse treatment on structure and hardness of cryorolled aluminum
}

\author{
I. Sh. Valeev, A. Kh. Valeeva ${ }^{\dagger}$, R. R. Ilyasov, E. V. Avtokratova, S. V. Krymskiy, \\ O. Sh. Sitdikov, M. V. Markushev \\ †valeevs@mail.ru
}

Institute for Metals Superplasticity Problems, RAS, Ufa, 450001, Russia

\begin{abstract}
The influence of the energy of electric pulse treatment (EPT) in the range of integral current densities $\left(K_{j}\right)$ from $0.06 \times 10^{5}$ to $0.29 \times 10^{5} \mathrm{~A}^{2} \mathrm{~s} / \mathrm{mm}^{4}$ on the structure and hardness of coarse-grained high-purity $\mathrm{Al}$, isothermally rolled up to a total strain of $90 \%$ at a liquid nitrogen temperature, was studied. It was found that EPT with an energy up to $K_{j}=0.104 \times 10^{5} \mathrm{~A}^{2} \mathrm{~s} / \mathrm{mm}^{4}$ practically did not affect the microhardness obtained in the cryorolled aluminum $(45-50 \mathrm{HV})$. An increase in the EPT energy to $K_{j}=0.121 \times 10^{5} \mathrm{~A}^{2} \mathrm{~s} / \mathrm{mm}^{4}$ led to a rapid drop of the hardness to $30 \mathrm{HV}$ followed by its gradual stabilization near $25 \mathrm{HV}$ at higher $K_{j}$ values. It was established that an enhanced microhardness of rolled Al resulted from formation of a well developed cellular structure with a crystallite size of about $2 \mu \mathrm{m}$, containing less than $10 \%$ of ultrafine grains of about $4 \mu \mathrm{m}$ in size. The minor hardness changes after EPT with $K_{j}$ up to $0.104 \times 10^{5} \mathrm{~A}^{2} \mathrm{~s} / \mathrm{mm}^{4}$ were related to occurrence of recovery and continuous recrystallization, resulted only in improving the deformation structure without noticeable changes in its type and the crystallite sizes. Therewith the softening caused by a partial decrease in the scalar dislocation density and the microdeformation of the crystal lattice was compensated by increasing the high angle boundaries fraction. At EPT with $K_{j}=0.121 \times 10^{5} \mathrm{~A}^{2} \mathrm{~s} / \mathrm{mm}^{4}$, the deformation structure was severely replaced by the fine-grain recrystallized one with the grain size of $19 \mu \mathrm{m}$, and resulted in the loss of the strenthening effect, caused by rolling. With further increase in the EPT energy, an extensive grain growth was observed, leading to the formation of a non-uniform structure grains and to appropriate material softening, owing to the grain coarsening. It was concluded that the restoration processes that took place during EPT were similar in nature to those that occur during furnace annealing of heavily deformed materials. Therewith, the short time of the thermal exposure on the deformed metal during EPT was compensated by high applied energies.
\end{abstract}

Keywords: cryogenic rolling, electrical pulse treatment, hardness, recrystallization, recovery.

УДК: 621.3.0.14.36

\section{Влияние электроимпульсной обработки на структуру и твердость криокатаного алюминия}

\author{
Валеев И.Ш., Валеева А. Х. ${ }^{\dagger}$, Ильясов Р. Р., Автократова Е. В., Крымский С. В., \\ Ситдиков О.Ш., Маркушев М.В.
}

Институт проблем сверхпластичности металлов РАН, Уфа, 450001, Россия

Исследовали влияние энергии электроимпульсной обработки (ЭИО) в интервале интегральных плотностей тока $\left(K_{j}\right)$ от $0.06 \times 10^{5}$ до $0.29 \times 10^{5} \mathrm{~A}^{2} \mathrm{c} / \mathrm{MM}^{4}$ на структуру и твердость крупнозернистого алюминия (Авч), изотермически прокатанного с суммарной степенью $90 \%$ при температуре жидкого азота. Обнаружено, что ЭИО с $K_{j}$ до $0.104 \times 10^{5} \mathrm{~A}^{2} \mathrm{c} /$ мм $^{4}$ практически не сказалась на твердости алюминия $(45-50 \mathrm{HV})$, достигнутой после криопрокатки. С увеличением энергии ЭИО до $K_{j}=0.121 \times 10^{5} \mathrm{~A}^{2} \mathrm{c} / \mathrm{MM}^{4}$ наблюдали резкое падение твердости до $30 \mathrm{HV}$ и последующую ее стабилизацию на уровне $25 \mathrm{HV}$ при больших значениях $K_{j}$. Установлено, что повышенная твердость алюминия после прокатки обусловлена структурным упрочнением вследствие формирования развитой ячеистой структуры с размером кристаллитов около 2 мкм, и содержащей не более 10\% (ультра)мелких зерен диаметром 4 мкм. После ЭИО с $K_{j}$ до $0.104 \times 10^{5} \mathrm{~A}^{2} \mathrm{c} / \mathrm{Mм}^{4}$ твердость слабо изменялась по причине прохождения процессов возврата и непрерывной рекристаллизации, приводящих лишь к совершенствованию нагартованной 
структуры без заметного изменения размера кристаллитов. При этом потеря твердости из-за уменьшения скалярной плотности дислокаций и микроискажений кристаллической решетки компенсировалась увеличением доли высоугловых границ. При ЭИО с $K_{j}=0.121 \times 10^{5} \mathrm{~A}^{2} \mathrm{c} / \mathrm{MM}^{4}$ деформационная структура алюминия активно замещалась рекристаллизованной мелкозернистой структурой с размером зерна 19 мкм, что сопровождалось потерей упрочняющего эффекта от прокатки. При дальнейшем повышении энергии отмечали интенсивный рост зерен и увеличение разнозернистости, приводившие к еще большему разупрочнению металла. Сделан вывод о том, что реализующиеся при электроимпульсном воздействии процессы, по природе и характеру близки к процессам, протекающим при печном отжиге сильнодеформированных материалов. При этом короткое время термического воздействия на деформированный алюминий компенсировалось высокими вносимыми энергиями ЭИО.

Ключевые слова: криогенная прокатка, электроимпульсная обработка, твердость, рекристаллизация, возврат.

\section{1. Введение}

Один из перспективных и сравнительно малоисследованных методов деформационного структурирования металлов и сплавов с целью придания им ультрамелкозернистого (УМЗ) и нанокристаллического строения (размер кристаллитов - зерен и субзерен, менее 1 и 0.1 мкм, соответственно) базируется на криогенных условиях обработки, т. е. деформации при температурах ниже $120 \mathrm{~K}\left(-153^{\circ} \mathrm{C}\right)$. В основе такого подхода лежит общеизвестное представление о том, что чем ниже температура деформирования, тем сильнее в обрабатываемом материале подавляются процессы динамического возврата, приводя к большему накоплению дефектов кристаллического строения и повышению внутренних напряжений [1-5]. При этом подразумевается то, что измельчение зерен и субзерен достигается активизацией как динамических (в процессе деформации, в особенности большой пластической деформации), так и постдеформационных, статических процессов. В результате криодеформирования и последующего термического воздействия, в обрабатываемом материале ожидалось формирование дисперсной и неравновесной структуры, обеспечивающей его повышенную прочность вследствие большего структурного упрочнения из-за дополнительного измельчения кристаллитов и более высокой их дефектности [3 - 7]. Однако, ряд проведенных экспериментальных исследований показал, что достижение указанных структурных состояний с использованием криогенной деформации не является столь простым и обязательным для всех металлов и сплавов. Так, для меди было установлено $[1,2]$, что снижение температуры ее деформирования ниже комнатной, на фоне упомянутого эффекта дополнительного упрочнения (нагартовки), подавляло двойное поперечное скольжение дислокаций и, тем самым, затрудняло формирование новых границ деформационного происхождения, замедляя фрагментацию структуры. А авторы [4-13] утверждают, что криогенные условия деформации априори не гарантируют интенсификацию процессов измельчения кристаллитов, в том числе, и процессов динамического наноструктурирования металлических материалов, включая чистые металлы с ГЦК решеткой.

Тем не менее, в литературе представлен и ряд более оптимистичных данных. Например, в [14-18] показано, что большая (интенсивная, мега, severe) пластическая деформация в криогенных условиях, в том числе реализованная листовой прокаткой, все же может обеспечить наноструктурирование металлов. При этом обработка должна быть двухстадийной, на второй стадии которой криодеформированные объекты предпочтительно подвергать высокоскоростному нагреву с кратковременной выдержкой. Такой нагрев обеспечивается, например, при электроимпульсной обработке (ЭИО), реализующей миллисекундное высокоэнергетическое термическое воздействие на обрабатываемый материал. Синергия двух экстремальных воздействий позволила, в частности, сформировать однородную УМЗ структуру в криокатанной меди $[14,15]$ и никеле $[16,17]$. При этом важнейшая роль в получении объектов с регламентированной дисперсностью структуры и повышенным комплексом свойств отводится режимам ЭИО, контролирующим полноту и кинетику процессов трансформации деформационной структуры в более равновесную, в том числе рекристаллизованную $[7,18]$.

Цель настоящей работы - оценить эффект ЭИО и энергии импульсов на структуру и твердость особочистого алюминия, деформированного прокаткой с обжатием 90\% при температуре жидкого азота.

\section{2. Материал и методы}

В качестве материала исследования использовали слиток алюминия марки Авч (Al 99.99\%) (ГОСТ 110692001). Слиток гомогенизировали при $400^{\circ} \mathrm{C}, 1$ ч, затем предварительно прокатывали при комнатной температуре до суммарной степени обжатия $75 \%$ и отжигали при $100^{\circ} \mathrm{C}, 10$ мин. Последующую криогенную деформацию механически вырезанных пластин толщиной 4 мм проводили на лабораторном шестивалковом стане в изотермических условиях, обеспечивавшихся предварительным охлаждением съемных рабочих валков диаметром 63 мм и заготовок в ванне с жидким азотом в течение 60 минут. При этом скорость прокатки не превышала 100 мм/мин, степень деформации (обжатия) за проход составляла 5 - 7\%, конечная толщина полос 0.4 мм. Из полос на электроэрозионном станке АРТА 207 вырезали образцы, имевшие форму образцов для механических испытаний с размерами рабочей части $3 \times 4 \times 0.4$ мм $^{3}$. Последующую ЭИО проводили по методике и с использованием оборудования, подробно описанным в [16]. Энергию обработки (интегральную плот- 
ность тока $\left.\left(K_{j}\right)\right)$ изменяли в диапазоне от $0.06 \times 10^{5}$ до $0.29 \times 10^{5} \mathrm{~A}^{2} \mathrm{c} / \mathrm{MM}^{4}$, что соответствовало расчетным температурам интервала нагрева образцов $70-550^{\circ} \mathrm{C}$. Вследствие того, что толщина образцов была в несколько раз меньше расчетной величины скин-слоя, считали проникновение тока однородным по их сечению. Для минимизации эффекта вылеживания при комнатной температуре на структуру и прочность криокатанного и термообработанных состояний, в интервале между всеми технологическими этапами их получения и анализа образцы хранили при температуре $-25^{\circ} \mathrm{C}$.

Микроструктуру исследовали в плоскости прокатки методом анализа дифракции обратно рассеянных электронов (EBSD) с использованием сканирующего электронного микроскопа «TESCAN MIRA 3 LMH» и программного комплекса «HKL Channel 5». Картины дифракции индицировали по 6 Кикучи-линиям при шаге сканирования 0.5 мкм. В качестве критерия разделения на мало- и высокоугловые границы (МУГ и ВУГ) использовали угол разориентировки $15^{\circ}$. Размеры зерен и субзерен $\left(d_{3}\right.$ и $d_{c з}$, соответственно) определяли методом эквивалентного диаметра. Средний угол разориентировки межкристаллитных границ $\left(\Theta_{\mathrm{cp}}\right)$ и долю высокоугловых границ $\left(F_{\text {вуг }}\right)$ определяли по данным разориентировок границ с $\Theta>2^{\circ}$. Долю рекристаллизованных зерен $\left(F_{\text {рек}}\right)$ рассчитывали как отношение площади рекристаллизованных зерен к площади всех зерен. Под рекристаллизованными понимали зерна с углами внутреннего искажения решетки менее $2^{\circ}$, и не содержащие субграницы.

Микротвердость определяли по методу Виккерса на приборе MVDM 8 «AFFRY» при нагрузке $0.1 \mathrm{H}$ и длительности ее приложения 10 с. Проводили не менее десяти измерений на точку, при этом ошибка измерений не превышала $5 \%$.

Рентгеноструктурный анализ (РСА) проводили на дифрактометре ДРОН-4-07 в $\mathrm{Cu}-\mathrm{K}_{\alpha}$ излучении при напряжении 40 кВ и токе 30 мА с длиной волны $\lambda=1.54418 \AA$ А. Съемку осуществляли методом пошагового сканирования (с шагом $0.1^{\circ}$ и временем экспозиции 4 сек) с вращением образца. Среднеквадратичную микродеформацию кристаллической решетки $\left(\left\langle\varepsilon^{2}\right\rangle^{1 / 2}\right)$ рассчитывали методом полнопрофильного анализа в программном комплексе «MAUD» с погрешностью не более $0.001 \%$. Плотность дислокаций, $\rho$, определяли как:

$$
\rho=2 \sqrt{3}\left\langle\varepsilon^{2}\right\rangle^{\frac{1}{2}} /(D \times b),
$$

где $b-$ вектор Бюргерса, $D-$ размер области когерентного рассеяния.

\section{3. Результаты и обсуждение}

Микроструктура алюминия после криопрокатки была неоднородной, преимущественно представлена вытянутыми фрагментами исходных зерен в виде волокон (Рис. 1), внутри которых наблюдали близкие к равноосной форме ячейки и/или рекристаллизованные зерна со средним размером 2 и 4 мкм, соответственно (Табл. 1). При этом доля рекристаллизованных зерен в волокнах варьировалась в диапазоне 2-10\% (в среднем около $3 \%$ ) при доле ВУГ порядка $11 \%$ и среднем угле разориентировки всех границ около $7^{\circ}$. Такая структура также характеризовалась высокими значениями скалярной плотности дислокаций и микроискажений решетки (Табл. 1), обеспечивавшими, наряду со структурным упрочнением (за счет измельчения кристаллитов), твердость алюминия (Рис. 2), превышавшую исходную почти в два раза.

Из Рис. 2 и 3, и данных в Табл. 1 следует, что параметры микроструктуры и твердости криокатанного алюминия сильно зависят от величины внесенной энергии ЭИО. Так, после ЭИО с энергией вплоть до $K_{j}=0.104 \times 10^{5} \mathrm{~A}^{2} \mathrm{c} / \mathrm{MM}^{4}$, его твердость оставалась практически на уровне деформированного материала (Рис. 2). При этом микроструктура (Рис. $3 \mathrm{a})$, как и после деформации, была смешанной, состоящей из областей мелких зерен и областей дислокационной ячеистой структуры с теми же средними размерами кристаллитов - зерен и субзерен (Табл. 1). Однако, плотность дислокаций и внутренние напряжения заметно уменьшались, а доли рекристаллизованных зерен и высокоугловых границ, как и средний угол разориентации границ, увеличивались.

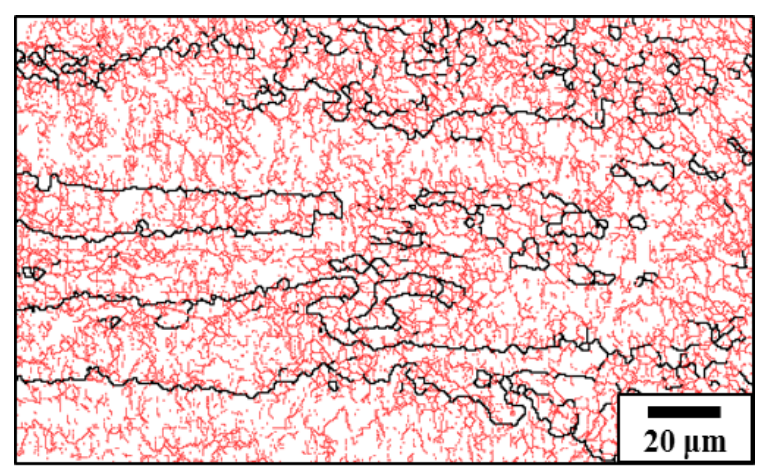

Pис. 1. (Color online) Микроструктура алюминия после криопрокатки с обжатием 90\% (здесь и далее ВУГ и МУГ окрашены черным и красным цветом, соответственно).

Fig. 1. (Color online) Microstructure of aluminum after cryorolling with reduction of $90 \%$ (here and after HABs and LABs are colored in black and red, consequently)

Табл. 1. Параметры структуры криодеформированного алюминия до и после ЭИО.

Table 1. Parameters of the structure of cryodeformed aluminum before and after EPT.

\begin{tabular}{|c|c|c|c|c|c|c|c|c|}
\hline \begin{tabular}{|c|}
$K_{j}, \times 10^{5} \mathrm{~A}^{2} \mathrm{c} / \mathrm{MM}^{4}$ \\
$K_{j}, \times 10^{5} \mathrm{~A}^{2} \mathrm{~s} / \mathrm{mm}^{4}$ \\
\end{tabular} & $\begin{array}{l}d_{\mathrm{c} 3}, \mathrm{MKM} \\
d_{\mathrm{sg}}, \mathrm{mm}\end{array}$ & $\begin{array}{l}d_{3}, \text { МКм } \\
d_{\mathrm{g}}, \mathrm{mm}\end{array}$ & $\begin{array}{l}F_{\text {pee }} \% \\
F_{\text {rec }} \%\end{array}$ & $\begin{array}{c}\Theta_{c p}, \text { град. } \\
\Theta_{\mathrm{av}}, \text { degree }\end{array}$ & $\begin{array}{l}F_{\text {Вуг }}, \% \\
F_{\mathrm{HAB}}, \%\end{array}$ & $\begin{array}{l}a, \AA \\
a, \AA\end{array}$ & \begin{tabular}{|c|} 
Плотность дислокаций, $10^{13} \mathrm{M}^{-2}$ \\
Dislocation density, $10^{13} \mathrm{~m}^{-2}$
\end{tabular} & $\begin{array}{l}\left\langle\varepsilon^{1 / 2}\right\rangle, \% \\
\left\langle\varepsilon^{1 / 2}\right\rangle, \%\end{array}$ \\
\hline- & $2 \pm 0.2$ & $4 \pm 0.4$ & 3 & 7 & 11 & $4.041 \pm 0.001$ & $4.8 \pm 0.5$ & $0.062 \pm 0.001$ \\
\hline 0.104 & $2 \pm 0.2$ & $4 \pm 0.8$ & 6 & 16 & 23 & $4.041 \pm 0.001$ & $1.8 \pm 0.3$ & $0.014 \pm 0.003$ \\
\hline 0.121 & $5 \pm 1$ & $19 \pm 6$ & 66 & 31 & 76 & $4.042 \pm 0.001$ & $0.1 \pm 0.1$ & $0.001 \pm 0.001$ \\
\hline 0.290 & $14 \pm 4$ & $53 \pm 14$ & 76 & 32 & 74 & $4.043 \pm 0.001$ & $0.1 \pm 0.1$ & $0.001 \pm 0.001$ \\
\hline
\end{tabular}




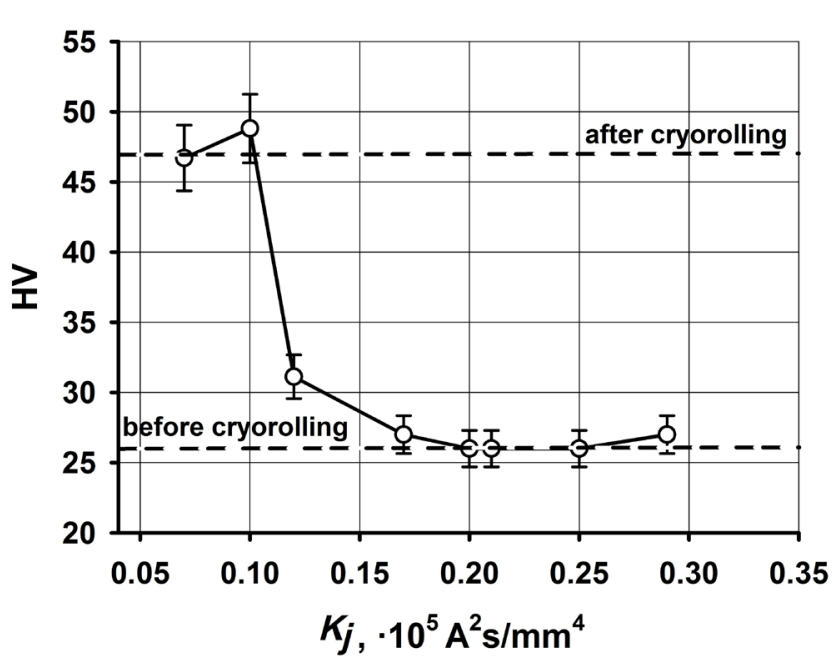

Рис. 2. Зависимость микротвердости криокатанного алюминия от величины энергии ЭИО.

Fig. 2. Microhardness of the cryorolled aluminum vs the EPT energy.

Этот результат, во-первых, указывал на то, что в областях деформационно-индуцированной субструктуры при ЭИО успевали проходить процессы статического возврата и/или непрерывной рекристаллизации по типу in-situ. Во-вторых, с этим же результатом была связана неизменность твердости алюминия. А именно, потеря твердости от фиксируемого уменьшения скалярной

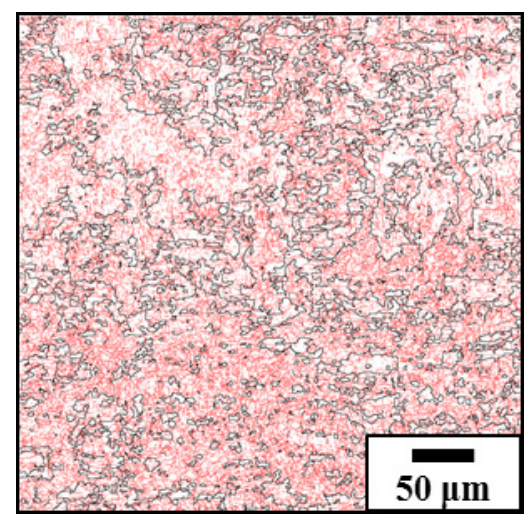

a

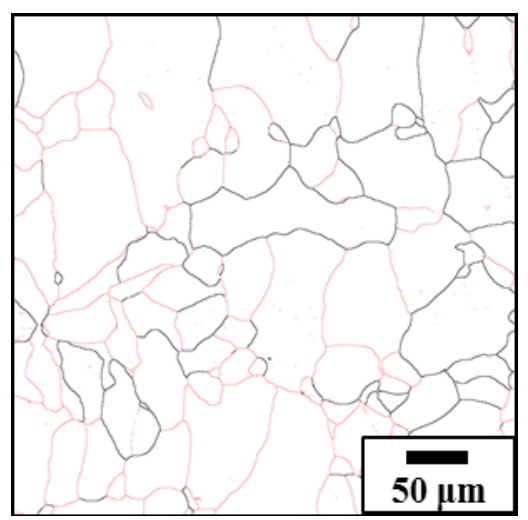

плотности дислокаций и микроискажений кристаллической решетки была скомпенсирована увеличенной долей новых зерен, т.е. увеличением вклада зернограничного упрочнения в соответствии с уравнением Холла-Петча [19]. Другим компенсирующим фактором могло послужить также то, что при указанных режимах ЭИО в материале могли развиваться процессы, приводившие к образованию новых «полигональных ячеек» (субзерен) вследствие перестройки дислокационной структуры. А границы последних являлись более эффективными барьерами для движения решеточных дислокаций, чем границы «деформационных ячеек» [20]. Следует отметить, что по расчетным данным максимальная температура разогрева образцов при $K_{j}=0.06 \times 10^{5}-0.104 \times 10^{5} \quad \mathrm{~A}^{2} \mathrm{c} / \mathrm{MM}^{4}$ находилась в диапазоне $70-130^{\circ} \mathrm{C}$, что приблизительно соответствовало пороговой температуре начала рекристаллизации алюминия высокой чистоты (около $100^{\circ} \mathrm{C}$ ) [21].

После ЭИО с $K_{j}=0.121 \times 10^{5} \quad \mathrm{~A}^{2} \mathrm{c} / \mathrm{MM}^{4}$ отмечалось резкое снижение твердости алюминия до $30 \mathrm{HV}$ (Рис. 2), сопровождаемое активной заменой деформационной структуры крупнозернистой рекристаллизованной с размером зерна около 20 мкм (Рис. 3b). Об этом прежде всего свидетельствовало резкое снижение практически до нулевых значений микроискажений решетки. Кроме того, помимо интенсивного роста зерен, низкой доли малоугловых границ и равновесной плотности дислокаций (Табл. 1), для полученной структуры были

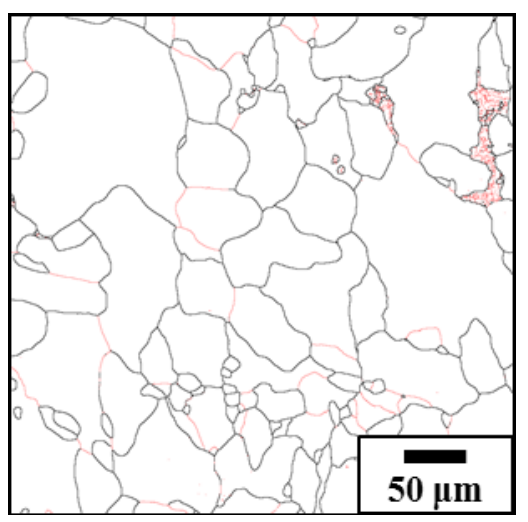

b

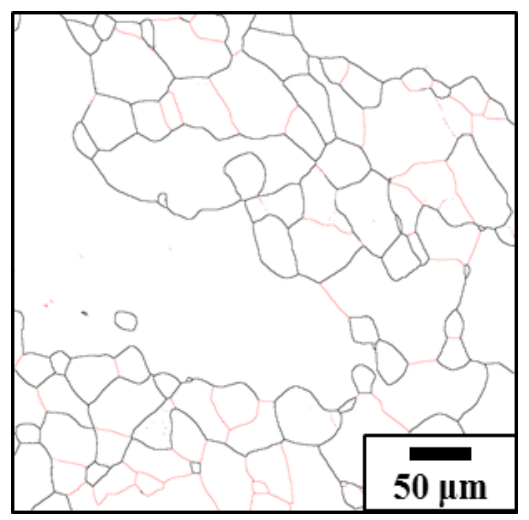

d

Pис. 3. (Color online) EBSD-карты межкристаллитных границ в алюминии после электроимпульсной обработки с энергиями $0.104 \times 10^{5}(\mathrm{a}), 0.121 \times 10^{5}(\mathrm{~b}), 0.172 \times 10^{5}$ (c) и $0.290 \times 10^{5} \mathrm{~A}^{2} \mathrm{c} / \mathrm{MM}^{4}(\mathrm{~d})$.

Fig. 3. (Color online) EBSD maps of intercrystallite boundaries in aluminum after electric pulse treatment with energies of $0.104 \times 10^{5}$ (a), $0.121 \times 10^{5}(\mathrm{~b}), 0.172 \times 10^{5}$ and $0.290 \times 10^{5} \mathrm{~A}^{2} \mathrm{~s} / \mathrm{mm}^{4}(\mathrm{~d})$. 
характерны искривленные границы и разнозернистость. По-видимому, причиной последней явилось наследование неоднородности деформированной структуры. Все отмеченные особенности структуры сохранялись и при больших энергиях ЭИО, и при этом имело место еще большее ее огрубление (Рис. 3 с и d).

Можно считать, что, как и в случае непрерывной статической рекристаллизации, наблюдаемой при печном отжиге сильнодеформированных металлов [22], в интервале $K_{j}=0.104 \times 10^{5}-0.172 \times 10^{5} \mathrm{~A}^{2} \mathrm{c} / \mathrm{Mм}^{4}$ основным процессом трансформации деформационной структуры криокатаного алюминия был «переходный» процесс от совершенствования структуры по типу in-situ, контролируемого возвратом, к нормальному росту зерна. Резкое падение твердости у левой границы интервала было связано с активизацией роста отдельных зерен, сформировавшихся при прокатке, которые потенциально могли выступать в качестве зародышей рекристаллизации. А способность к быстрому росту отдельных зерен, формирующихся in-situ в деформированной структуре, могла быть также обусловлена структурной неоднородностью, присущей деформационному состоянию, и различной кинетикой возврата в каждом зерне. Таким образом, при определенной величине внесенной энергии возврат «высвобождал» часть кристаллитов от дефектов и обеспечивал их способность к росту, тогда как другие кристаллиты могли быть все еще «заняты» дислокациями высокой плотности и претерпевать полигонизацию. При этом рост отдельных зерен являлся следствием миграции их границ, которая проходила под действием движущих сил, обусловленных предшествующей деформацией. Во время миграции граница растущего зерна поглощала дефекты кристаллического строения: вакансии, дислокации, другие границы [19] и вызывала изменения внутренней энергии системы и, как следствие, - снижение твердости, приобретенной при прокатке.

Стоит также отметить, что границы новых зерен, имевшие большую удельную энергию после больших степеней криодеформации, были способны к быстрому перемещению при ЭИО. В отличие от таких границ, энергия и разоринтация границ как динамически, так и статически сформированных ячеек, были меньше. Поэтому последние были менее подвижны, и мигрировали с меньшими скоростями, чем границы рекристаллизованных зерен. Интенсивный рост зерен был обусловлен и тем, что чистый алюминий не содержал вторых фаз, способных существенно повлиять на его структурное поведение при ЭИО. В результате, при малых энергиях (температурах нагрева) фиксировали лишь миграцию границ рекристаллизованных зерен, осуществлявшуюся в сторону деформированной матрицы. А при высоких энергиях после исчезновения деформированной структуры (по окончании перехода от процессов рекристаллизации и полигонизации к нормальному росту зерен), границы зерен продолжали мигрировать под действием движущей силы, вызванной уменьшением локального радиуса кривизны границ при уменьшении их протяженности, что сопровождалось увеличением размера зерна (Табл. 1, Рис. 3).
Таким образом, можно заключить, что процессы, реализовавшиеся при ЭИО криокатаного алюминия, близки по природе и характеру к процессам, протекающим при статическом отжиге сильнодеформированных материалов - статическому возврату, непрерывной статической рекристаллизации и росту зерна. При этом короткое время (импульсный характер) термического воздействия на деформированный металл компенсировалось высокими вносимыми энергиями (температурами) ЭИО. Так как возврат и статическая рекристаллизация контролируются самодиффузией [22], в соответствии с законом Аррениуса даже небольшое повышение температуры (энергии ЭИО) должно было приводить к резкому повышению скорости этих процессов и, соответственно, снижению времени их завершения.

\section{4. Заключение}

Установлено, что однократная электроимпульсная обработка криокатаного алюминия Авч, проводимая с энергий, ниже «порогового» значения $K_{j}$, составляющего $0.104 \times 10^{5} \mathrm{~A}^{2} \mathrm{c} / \mathrm{MM}^{4}$, приводит к развитию преимущественно статического возврата и активизации in-situ непрерывной статической рекристаллизации с характерными минимальными качественными и количественными структурными изменениями и сохранением твердости, фиксируемой после прокатки. ЭИО с превышением указанного порогового значения энергий активизирует рекристаллизационные процессы, обеспечивая переход от рекристаллизации in-situ к росту зерен, что приводит к резкому снижению твердости. Дальнейшее увеличение энергии ЭИО до $0.290 \times 10^{5} \mathrm{~A}^{2} \mathrm{c} / \mathrm{Mм}^{4}$ приводит к формированию крупнозернистой структуры с размером зерна более 50 мкм и стабилизации твердости на уровне недеформированного металла.

Благодарности /Acknowledgements. Работа выполнена при поддержке гранта РФФИ №19-08-00953А. Экспериментальные исследования были выполнены на базе Центра коллективного пользования ИПСМ РАН "Структурные и физико-механические исследования материалов». / The work was supported by the RFBR under grant No. 19-08-00953A. The work was performed using the facilities of the shared services center "Structural and PhysicalMechanical Studies of Materials" at the Institute for Metals Superplasticity Problems of Russian Academy of Sciences.

\section{Литература/Referencies}

1. I. A. Gindin, M.B. Lazareva, V.P. Lebedev, Ya.D. Starodubov. FMM. 23, 138 (1967). (in Russian) [И.А. Гиндин, М.Б. Лазарева, В.П. Лебедев, Я.Д. Стародубов. ФММ. 23, 138 (1967).]

2. I.A. Gindin, M. B. Lazarev, V.P. Lebedev et al. FMM. 24, 347 (1967). (in Russian) [И.А. Гиндин, М.Б. Лазарева, В. П. Лебедев и др. ФММ. 24, 347 (1967).]

3. Y. Huang, P. B. Prangnell. Acta Mater. 56, 1619 (2008). Crossref 
4. S. V. Krymskiy, E.V. Avtokratova, O.S. Sitdikov, A. V. Mikhaylovskaya, M. V. Markushev. Physics of Metals and Metallography. 116 (7), 676 (2015). $\underline{\text { Crossref }}$

5. S. Krymskiy, O. Sitdikov, E. Avtokratova, M. Markushev. Transactions of Nonferrous Metals Society of China (English Edition). 30 (1), 14 (2020). Crossref

6. D. Magalhaes, A. Kliauga, M. Ferrante, V. Sordi. J. Mater. Sci. 52, 7466 (2017). Crossref

7. M. Markushev, S. Krymskiy, R. Ilyasov, E. Avtokratova, A. Khazgalieva, O. Sitdikov. Lett. Mater. 7 (4), 447 (2017). Crossref

8. J. Shi, L. Hou, J. Zuo, L. Zhuang, J. Zhang. Materials Science \& Engineering A. 701, 274 (2017). Crossref

9. S. Choi, J. Won, S. Lee, J. Hong, Y. Choi. Materials Science \& Engineering A. 738, 75 (2018). Crossref

10. V.S. Sarma, J. Wang, W. W. Jian, A. Kauffmann, H. Conrad, J. Freudenberger, Y.T. Zhu. Mater Sci Eng A. 527, 7624 (2010). Crossref

11. T. Konkova, S. Mironov, A. Korznikov, S.L. Semiatin. Acta Mater. 58, 5262 (2010). Crossref

12. I.Sh. Valeev, Z.G. Kamalov. JMEPEG. 12, 272 (2003). Crossref

13. L. Voronova, M. Degtyarev, T. Chashchukhina, T. Gapontseva, V. Pilyugin. Lett. Mater. 8 (4), 424 (2018). Crossref
14. T. Konkova, I. Valeev, S. Mironov et al. J. All. Comp. 659, 184 (2016). Crossref

15. T. Konkova, I. Valeev, S. Mironov et al. J. Mater. Res. 29 (22), 2727 (2014). Crossref

16. I. Sh. Valeev, A. Kh. Valeeva, R. R. Ilyasov, O. Sh. Sitdikov, M. V. Markushev. Lett. Mater. 9 (4), 447 (2019). Crossref

17. R. R. Ilyasov, A. Kh. Valeeva, I. Sh. Valeev, O. Sh. Sitdikov, M. V. Markushev. IOP Conf. Series: Materials Science and Engineering. 1008, 012006 (2020). Crossref

18. Y. Sheng, Y. Hua, X. Wang, X. Zhao, L. Chen, H. Zhou, J. Wang, C.C. Berndt, W.Li. Materials. 11, 185 (2018). Crossref

19. F. J. Humphreys, M. Hatherly. Recrystallization and Related Annealing Phenomena. Elsevier (2004) 658 p. Crossref

20. A. M. Russell, K.L. Lee. Stucture-Property Relations in Nonferrous Metals. New York, Wiley (2005) 440 p. Crossref

21. S. S. Gorelik. Recristallizatsiya metallov i splavov. Moscow, Metallurgiya (1978) 568 p. (in Russian) [C. С. Горелик Рекристаллизация металлов и сплавов. Москва, Металлургия (1978) 568 с.]

22. A. Belyakov, T. Sakai, H. Miura, R. Kaibyshev, K. Tsuzaki. Acta Materialia. 50 (6), 1547 (2002). rossref 